\title{
Cosmic Shear in 3D
}

\author{
Alan F. Heavens ${ }^{1}$, Thomas D. Kitching ${ }^{1}$ \\ ${ }^{1}$ Institute for Astronomy, University of Edinburgh, Blackford Hill, Edinburgh EH9 3HJ, U.K. \\ email: afh@roe.ac.uk, tdk@roe.ac.uk
}

\begin{abstract}
Cosmic shear surveys are now collecting photometric redshifts of the sources. We analyse how such photometric redshifts can be used to perform a genuine 3D analysis of the shear fields, with enhanced statistical power over conventional shear analysis on the sky. In particular, 3D lensing has the potential to measure accurately the equation of state of Dark Energy, with a possibility of measuring $w$ with an accuracy of a few percent. Like the microwave background, 3D lensing observables are calculable $a b$ initio, and are largely unaffected by complex astrophysical processes which may limit their power. In this context, we review how 3D information from photometric redshifts can reduce a possible physical systematic effect, arising from intrinsic alignments of galaxies.
\end{abstract}

\section{Introduction}

Cosmological parameter estimation has advanced tremendously with the combination of highly successful cosmic microwave background (CMB) experiments such as WMAP and CBI with other cosmological datasets such as the galaxy surveys from $2 \mathrm{dF}$ and SDSS. One can trace this success to several facts: firstly, the CMB observables can be predicted robustly $a b$ initio for given cosmological models; secondly, those observables have high sensitivity to the parameters; finally, although the observables are contaminated by systematics, both instrumental and from foreground sources, these can be controlled or eliminated to high accuracy. We argue here that weak lensing shares the same advantages, and, with the important addition of 3D shear information, can be used to perform precision cosmology beyond what has been achieved with the CMB.

The main scientific goal of the next generation of cosmological experiments comes from the remarkable conclusion of current studies that the Universe is dominated by Dark Energy and Dark Matter. The determination of the properties and nature of these components will be the major focus for cosmological studies in the future. The CMB is not a sensitive probe of these components, but weak gravitational lensing on a cosmic scale is.

As with the CMB, cosmic shear exhibits desirable characteristics: the physics is simple, and observables can be predicted very robustly for cosmological models. In particular, as we show here, the use of 3D cosmic shear methods adds considerable statistical power for the determination of the properties of Dark Energy (characterised by $w$, where $P=w \rho c^{2}$ ), potentially measuring $w(z)$ with high precision, with statistical errors of a few percent being achievable. There remains a question of whether the theoretical precision can be achieved, and this will in future be determined by systematic errors. These systematics can be broadly divided into those which are concerned with the optical quality of the images, and 'physical' systematics, arising from the contamination of the lensing signal by astrophysical effects. In many other fields, fundamental limits on what one can learn may come from an incomplete understanding of complex physical systems, such as galaxies or clusters of galaxies. Ultimately, there is no guarantee that such uncertainties 
will ever go away. For cosmic shear, where one interprets the alignment of images as an indication of intervening clumpy mass distribution, the intrinsic alignment of galaxies can mimic a lensing signal. The intrinsic alignment of galaxies is a complex physical issue, but we review below how this potential limiting systematic can be removed.

\section{Shear, Dark Matter and Cosmology}

The cosmic shear field $\gamma=\gamma_{1}+i \gamma_{2}$ can be estimated at the positions of source galaxies from their ellipticities. Weak lensing induces correlations in the ellipticities of the galaxy images, correlations whose detailed properties depend on cosmological parameters. In 2D studies of cosmic shear, a typical statistic used is the shear correlation function on the sky; the lensing contribution to this can be calculated in terms of the power spectrum of the matter fluctuations: for galaxies separated on the sky by an angle $\theta$,

$$
\langle\gamma \gamma *\rangle(\theta)=\frac{1}{2 \pi} \int d s s P_{\kappa}(s) J_{0}(s \theta)
$$

where the convergence power spectrum is

$$
P_{\kappa}(s)=\frac{9}{4}\left(\frac{H_{0}}{c}\right)^{4} \Omega_{m}^{2} \int d r n^{2}(r)(1+z)^{2} P_{\text {mass }}(s / r) .
$$

$H_{0}$ is the Hubble constant, $\Omega_{m}$ is the present-day matter density parameter, and $n(r)$ is the number density of sources as a function of radial coordinate $r(z)$. Thus the shear correlation function is sensitive to cosmological parameters through a number of effects: the shape of the linear matter power spectrum; the nonlinear evolution of $P_{\text {mass }}$, and the $r(z)$ relation. It is important to note that the accurate determination of cosmological parameters requires an accurate redshift distribution of the sources. This has motivated the acquisition of photometric redshifts for cosmic shear surveys, and all current and planned future surveys will obtain photometric redshifts.

\section{Photometric redshifts}

Although the initial motivation for obtaining photometric redshifts was an accurate determination of $n(z)$, surveys with photometric redshifts offer two further substantial advantages. One of these concerns the elimination of a potentially limiting physical systematic; the second is a statistical advantage in using the full $3 \mathrm{D}$ positional information of the sources.

\section{Physical Systematics: intrinsic alignments}

The shear is estimated from the ellipticity of source galaxies; in the weak shear case, the observed ellipticity is related to the shear and the intrinsic ellipticity $e_{I}$ by $e=e_{I}+2 \gamma$. Hence the ellipticity correlation is

$$
\left\langle e e^{*}\right\rangle=4\left\langle\gamma \gamma^{*}\right\rangle+\left\langle e_{I} e_{I}^{*}\right\rangle+4\left\langle e_{I} \gamma^{*}\right\rangle .
$$

If the last two terms are zero, the ellipticity correlation function is an unbiased estimate of the shear correlation function. However, studies in recent years (e.g. Heavens, Refregier \& Heymans (2000), Croft \& Metzler (2000), Crittenden et al. (2001), Catelan et al. (2001)) have shown that the intrinsic alignment signal $\left\langle e_{I} e_{I}^{*}\right\rangle$ does not vanish and could be an important source of systematic error if it is not dealt with. It could, in principle, limit the fundamental accuracy with which cosmic shear surveys can estimate parameters. Theoretically alignment of close pairs is expected at some level, as a result of tidal torques, but the level of alignment is hard to calculate, as nonlinear gravitational effects 


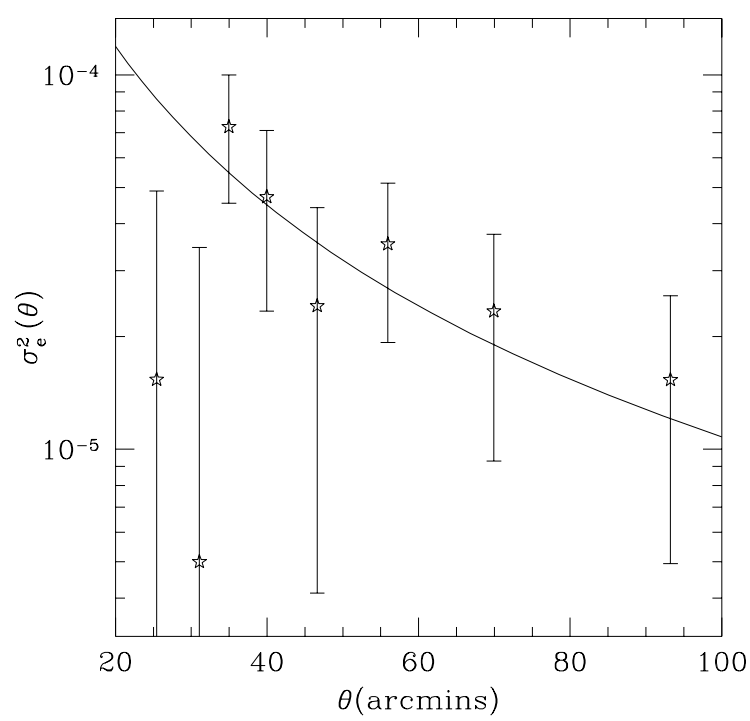

Figure 1. The ellipticity correlation function derived from the SuperCOSMOS survey (Brown et al. 2002; data) and the theoretical model of Heavens, Refregier \& Heymans (2000), modified to include an offset between baryon and dark matter angular momenta (Heymans et al 2004).

are likely to be involved, and numerical simulations require simultaneously very good mass resolution and a large volume. Nevertheless, a combination of theoretical studies and observational data from the low-redshift SuperCOSMOS survey (Brown et al. (2002)) have indicated the likely level of intrinsic alignments. The intrinsic ellipticity correlation function from SuperCOSMOS and from the theoretical model of Heavens, Refregier \& Heymans (2000), modified to allow for a misalignment of the baryon and dark matter components, is shown in Fig.1. The agreement is very good, but sufficient uncertainties remain that it is preferable to devise statistics which avoid the contamination if possible, rather than to try to model the intrinsic alignment effect and subtract it from the signal. At the expense of slightly increased shot noise, the issue can be essentially avoided altogether: photometric redshifts can be used to remove or downweight pairs in any estimate of a quadratic quantity such as the ellipticity correlation function. As shown by Heymans and Heavens (2003) and King \& Schneider (2002), this can reduce the intrinsic contamination to a negligible level. This pair removal has been done for the COMBO-17 survey (Heymans et al. (2004)), and shows a small systematic reduction in the estimate of the amplitude of mass clustering (through $\sigma_{8}$ ). As a result of the effectiveness of pair removal, the intrinsic alignment of galaxies should not be a fundamental limitation on the accuracy of cosmic shear surveys.

The last term, $\left\langle e_{I} \gamma^{*}\right\rangle$ has recently received some interest (Hirata \& Seljak (2004)); it arises from tidal effects potentially aligning a foreground galaxy, and simultaneously causing a mass distortion which leads to an aligned shear of the background source images. At this stage, the amplitude of this effect is not well known, but it may be important as the pair removal scheme outlined above will not remove this systematic. See Uros Seljak's contribution to these proceedings. 


\section{3D Cosmic Shear}

Evidently if one has some distance information for individual sources in a cosmic shear survey, it makes sense to use it. The 3D information allows us to construct lensing observables with considerably enhanced statistical power over the $2 \mathrm{D}$ counterparts. The most exciting prospect is to measure the properties of the Dark Energy. Often characterised by the equation of state parameter $w$, where $p=w \rho c^{2}$, the crucial questions are whether $w=-1$ (in which case the dark energy may be Einstein's cosmological constant, i.e. a feature of gravity theory rather than a vacuum energy), and if not, what its value is and whether it evolves with redshift. More generally, 3D weak lensing may well offer the most powerful tests of theories of Dark Energy.

A straightforward way of including 3D information is to divide the sources into bands, based on their photometric redshifts, and to perform a 2D analysis of each slice (and/or consider cross-correlations between slices). This tomography, or ' $2 \frac{1}{2} D$ ' analysis, can be effective in reducing error bars (see e.g. Hu 1999), but it is not extracting everything from the data, and it is sensible to perform a full $3 \mathrm{D}$ analysis.

With photometric redshifts, one has a set of estimates of the shear at locations in $3 \mathrm{D}$, albeit with imprecise distance estimates. A couple of approaches are possible, either based on the correlation of shears in 3D (see David Bacon's article in these proceedings), or a power spectrum approach, which is advocated here. The main motivation for this latter approach is that even in $2 \mathrm{D}$ it is evident from equation 2.2 that it is necessary to have robust predictions for the mass power spectrum. In $3 \mathrm{D}$ this is still the case, and at high wavenumbers, these predictions become increasingly difficult. To get accurate results from weak lensing surveys, it is necessary to probe scales where nonlinearities have modified the shape and amplitude of the power spectrum. The matter power spectrum can be accurately modelled well into the nonlinear regime (Smith et al. (2003)), but at some point on small scales the effects of finite mass resolution, or physical effects such as the complex behaviour of the baryon component (e.g. White (2004)), modify the power spectrum in a way which is difficult to compute reliably. This motivates using an analysis method which is based in $k$-space rather than configuration space, so modes which are too strongly influenced by very small scales can be excluded from analysis. In Heavens (2003) we proposed a method based on spherical harmonics and spherical Bessel functions, which is particularly suited to wide-angle surveys. Here we propose a smallangle version, appropriate for small patches of sky. Full details will appear in Heavens et al. (2004).

The two shear fields $\gamma_{1}$ and $\gamma_{2}$ can be estimated at the positions of the galaxies in the sample. We consider a transformation of these shear fields, using spherical coordinates as a basis. The advantages of this system, rather than using a Cartesian system and associated Fourier transform, are several: the lensing effect is a radial effect, determined by the potential along the line of sight (assuming the Born approximation); the photometric redshifts give radial errors in position; the survey is specified normally by a sky coverage (angular) and a (radial) selection function. As a basis for the transform, we use products of spherical Bessel functions and exponentials. The latter are small-angle combinations of spherical harmonics; the choice of spherical harmonics and spherical Bessel functions is motivated by the fact that, as eigenfunctions of the Laplace operator, they allow a simple relationship between the lensing potential coefficients and the mass density coefficients. This allows the covariance matrix of the lensing coefficients to be readily related to the 
mass power spectrum. The transforms we perform are

$$
\hat{\gamma}_{i}(k, \ell) \equiv \sqrt{\frac{2}{\pi}} \sum_{\text {galaxies g }} \gamma_{i}\left(\mathbf{r}_{g}\right) j_{\ell}\left(k r_{g}^{0}\right) \exp \left(-i \ell \cdot \theta_{g}\right) W\left(r_{g}^{0}\right) \quad i=1,2 .
$$

$k$ is a radial wavenumber, and $\ell$ a $2 \mathrm{D}$ angular wavenumber. Thus the expansion is fully 3D. $W(r)$ is an arbitrary radial weighting which will not be explored here; the results we show will have $W=1 . \theta_{g}$ and $r_{g}^{0}$ are the angular and radial coordinates of a galaxy, the latter based on the photometric redshift of the galaxy, and assuming some fiducial set of cosmological parameters. The shear field is, however, the shear field at the actual radial coordinate of the galaxy, denoted by $r_{g}$. The transform is intended to be done once; since the fiducial model is in general wrong, the analysis takes this into account in computing the likelihood of the actual cosmological parameters. The coefficients of the shear expansion are related to the coefficients of the mass power spectrum, $\delta(k, \ell ; r)$ by (we quote only $\gamma_{2}$; note the time-dependence of the power spectrum is indicated by an $r$-dependence)

$$
\gamma_{2}(k, \ell) \propto-\ell_{x} \ell_{y} H_{0}^{2} \Omega_{m} \int_{0}^{\infty} d z d z_{p} p\left(z_{p} \mid z\right) \bar{n}\left(z_{p}\right) j_{\ell}(k r) W(r) I_{\ell}(r[z], k, \ell)
$$

where

$$
I_{\ell}(r, k, \ell)=\int_{0}^{r} d r^{\prime}\left(\frac{1}{r}-\frac{1}{r^{\prime}}\right)\left(1+z^{\prime}\right) \int d k^{\prime} j_{\ell}\left(k^{\prime} r^{\prime}\right) \delta\left(k^{\prime}, \ell ; r^{\prime}\right) .
$$

Here, $p\left(z_{p} \mid z\right)$ is the probability of a photometric redshift $z_{p}$ given a true redshift $z$, and a flat Universe has been assumed. We see that this expression demonstrates the integral effect of lensing, and includes arbitrary photometric redshift errors.

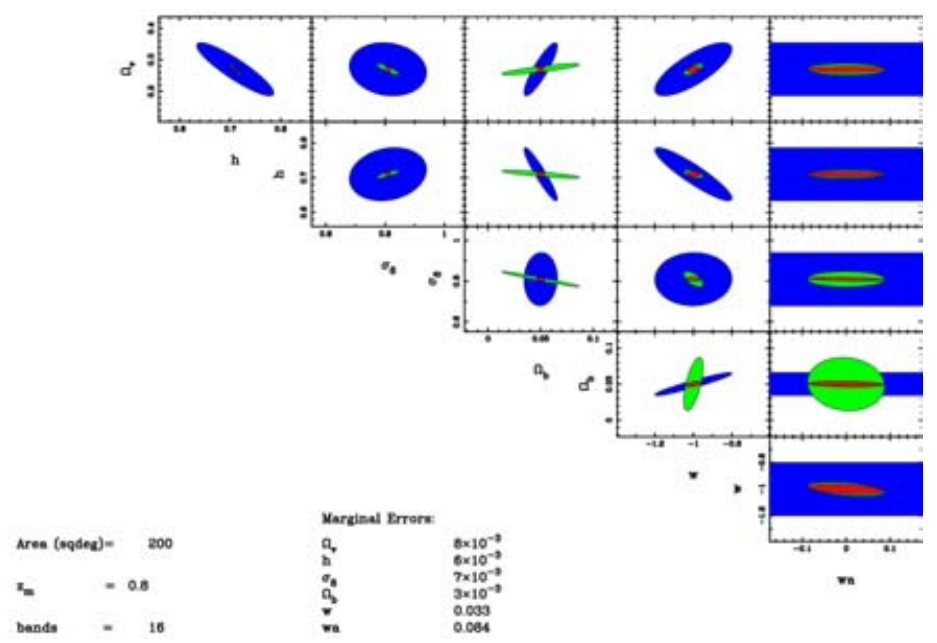

Figure 2. The expected error ellipses for cosmological parameters (dark energy density parameter, Hubble parameter $/ 100 \mathrm{~km} \mathrm{~s}^{-1} \mathrm{Mpc}^{-1}, \sigma_{8}$, baryon density parameter and dark energy equation of state $w$ ) from a lensing survey of 300 square degrees, with a median redshift of 1 and a photometric redshift error of $\sim 0.03$. Probabilities are marginalised over all other parameters, except that $n=1$, constant $w$ and a flat Universe are assumed. Dark shows a WMAP prior, light shows the $3 \mathrm{D}$ lensing constraints, and the central ellipses show the combination. 
These coefficients have zero mean, and a covariance which is related to the matter power spectrum, via $\left\langle\delta(k, \ell ; r) \delta^{*}\left(k^{\prime}, \ell^{\prime} ; r\right)\right\rangle \propto P_{\text {mass }}(k ; r) \delta^{D}\left(k-k^{\prime}\right) \delta^{D}\left(\ell-\ell^{\prime}\right) / k^{2}$, where $\delta^{D}$ is the Dirac delta function. With the addition of shot noise, the full covariance matrix is known, and standard likelihood methods can be used to determine cosmological parameters.

Figure 2 shows preliminary Fisher matrix calculations of the expected errors in cosmological parameters from a survey of 200 square degrees, with a median redshift of unity, and a photometric redshift error of 0.03 (typical of what can be achieved with a COMBO-17 type survey with c. 17 bands). These errors are marginalised over other cosmological parameters, and include a CMB prior. We see that errors of a few percent on $w$ are achievable, and this may improve as we analyse a wider range of 3D modes.

\section{Conclusions}

We have shown here that 3D analysis of cosmic shear offers two significant material advantages over $2 \mathrm{D}$ studies. Firstly, there is much greater statistical power for determining the equation of state of Dark Energy, and secondly, 3D information from photometric redshifts allows one to eliminate a potentially limiting systematic contamination of the lensing signal by the intrinsic alignment of galaxies. Given that the $3 \mathrm{D}$ lensing observables are calculable robustly $a b$ initio, and complex astrophysical complications avoidable, 3D cosmic shear offers the prospect of furthering cosmological studies beyond what can be done with the CMB alone, but with the same robust conclusions. Cosmic shear studies are in their infancy, but the medium-term prospects are excellent, with the potential to measure the Dark Energy equation of state parameter $w$ to an accuracy of a few percent.

\section{References}

Brown M.L., Taylor A.N., Hambly, N.C., Dye S. 2002, MNRAS 333, 501-509.

Catelan P., Kamionkowski M., Blandford R. D. 2001, MNRAS 320 L7-L13.

Crittenden R.G., Natarajan P., Pen U.-L., Theuns T. 2001, ApJ 559, 552-571.

Croft, R. A. C., Metzler, C. A. 2000, ApJ 545, 561-571.

Heavens A.F. 2003, MNRAS 343, 1327-1343.

Heavens A.F., Refregier A., Heymans C.E.C. 2000, MNRAS 319, 649-656.

Heavens A.F. et al. 2004, in preparation.

Heymans C.E.C., Heavens A.F. 2003, MNRAS 339, 711-720.

Heymans C.E.C., Brown M., Heavens A.F., Meisenheimer K., Taylor A.N., Wolf C. 2004, MNRAS 347, 895-908.

Hirata C., Seljak U. 2004, astroph 0416275.

King L., Schneider P. 2002, A\&A 396, 411-418.

Smith R. E., Peacock J. A., Jenkins A., White, S. D. M., Frenk C. S., Pearce F. R., Thomas P. A., Efstathiou G., Couchman H. M. P. 2003, MNRAS 341, 1311-1332.

White M. 2004, astroph 0405593. 